\title{
Ultrastructural Correlates of Quantal Synaptic Function at Single CNS Synapses
}

\author{
Paul J. Mackenzie, ${ }^{1}$ Gail S. Kenner, ${ }^{1}$ Oliver Prange, ${ }^{1}$ Hossein Shayan, ${ }^{1}$ Masashi Umemiya, ${ }^{3}$ and \\ Timothy H. Murphy ${ }^{1,2}$ \\ Kinsmen Laboratory of Neurological Research, Departments of ${ }^{1}$ Psychiatry and ${ }^{2}$ Physiology, University of British \\ Columbia, Vancouver, British Columbia V6T 1Z3, Canada, and '3Department of Neurophysiology, Tohoku University \\ School of Medicine, Sendai 980-8575, Japan
}

\begin{abstract}
We have tested the hypothesis that functional differences between synapses are associated with ultrastructure in cultured cortical neurons. Using $\mathrm{Ca}^{2+}$ imaging, we measured NMDA receptor-mediated miniature synaptic calcium transients attributed to the spontaneous release of single transmitter quanta. After imaging, the identified neurons were processed for serial transmission electron microscopy. At sites of quantal NMDA receptor-dependent $\mathrm{Ca}^{2+}$ transients, we confirmed the presence of excitatory synapses and measured spine size and
\end{abstract}

Studies using electron microscopy (EM) have indicated that the morphology of cortical excitatory glutamatergic synapses is highly variable (Harris et al., 1992). Bouton size, vesicle number, vesicle diameter, and postsynaptic spine volume vary widely within a relatively homogenous population of neurons (Pierce and Lewin, 1994; Schikorski and Stevens, 1997). It has been hypothesized that structural differences between synapses underlie some of the functional differences that are observed between neurons (Calverley and Jones, 1990; Lisman and Harris, 1993; Edwards, 1995). Accordingly, it is necessary to assess both synaptic structure and function at single CNS synapses. A few reports have combined EM and paired cell electrophysiology to measure the properties of single synapses (Gulyás et al., 1993; Buhl et al., 1994, 1997), but these experiments could not compare multiple synapses within a single neuron. To compare multiple synapses along a region of dendrite, we have measured spontaneous synaptic events using $\mathrm{Ca}^{2+}$ imaging at identified synapses in cultured cortical neurons and subsequently processed the same specimens for serial transmission EM. $\mathrm{Ca}^{2+}$ transients in individual spines can be resolved in an acute brain slice (Petrozzino et al., 1995; Yuste and Denk, 1995; Schiller et al., 1998; Yuste et al., 1999), but subsequent identification of the same synapses with EM would be problematic because of the higher spine density in the slice. Also, the three-dimensional (3-D) nature of neuronal dendrites in situ, as opposed to more planar dendrites found in culture, makes simultaneous imaging of multiple synapses within a slice difficult.

\footnotetext{
Received Dec. 14, 1998; revised March 29, 1999; accepted April 7, 1999.

This work was supported by grants from the Medical Research Council of Canada, EJLB foundation, and the British Columbia Health Research Fund (to T.H.M.). T.H.M. is an EJLB and Medical Research Council scholar. P.J.M. is supported by a Medical Research Council scholarship. O.P. is supported by a Deutscher Akademischer Austavschdienst and Heinrich-Hertz scholarship. We thank D. Brink, M. VonKrosigk, and C. Isbister for helpful comments on this manuscript.

Correspondence should be addressed to Timothy H. Murphy, Kinsmen Laboratory of Neurological Research, University of British Columbia, Vancouver, British Columbia V6T 1Z3, Canada.

Copyright (C) 1999 Society for Neuroscience $\quad 0270-6474 / 99 / 190001-\bullet \$ 05.00 / 0$
}

synaptic contact area. Our results demonstrate that synapse size correlates positively with the amplitude of the NMDA receptor-mediated postsynaptic response, suggesting that larger synapses express a greater number of NMDA receptors. Therefore, regulation of quantal amplitude may involve processes that alter synapse size.

Key words: NMDA; quantal; spine; dendrite; postsynaptic density; PSD; LTP

Using $\mathrm{Ca}^{2+}$ imaging, we have measured the NMDA receptormediated component of spontaneous miniature EPSCs (mEPSCs), termed the miniature synaptic $\mathrm{Ca}^{2+}$ transient, (MSCT; Murphy et al., 1994, 1995). Sensitivity to the NMDA receptor antagonist DL-APV suggests that under the conditions we have used, $\mathrm{Ca}^{2+}$ transients associated with miniature synaptic activity are primarily attributed to NMDA receptors (Murphy et al., 1994). We have previously reported a positive correlation between MSCT amplitude and mEPSC amplitude (Murphy et al., 1995), indicating that $\mathrm{Ca}^{2+}$ imaging can be used to evaluate the local characteristics of synaptic events. After MSCT imaging, we performed serial reconstruction of transmission EM images to identify synapses at the origins of the $\mathrm{Ca}^{2+}$ transients. This enabled the ultrastructural characterization of the specific synapses where MSCTs were measured. We show that MSCT amplitude is positively correlated with attributes of synapse size, including synaptic contact area and spine volume.

\section{MATERIALS AND METHODS}

Cortical neurons and glia were dissociated from 17-18 d gestation rat fetuses, placed in culture, and allowed to mature for $17-26 \mathrm{~d}$ in vitro as previously described (Mackenzie et al., 1996). Whole-cell recording was used to load neurons with fluo-3 and furaptra. The patch pipette solution contained (in $\mathrm{mm}$ ): $3-5$ fluo- $3 \mathrm{~K}^{+}$salt, 5 furaptra $\mathrm{K}^{+}$salt (relatively

This article is published in The Journal of Neuroscience, Rapid Communications Section, which publishes brief, peerreviewed papers online, not in print. Rapid Communications are posted online approximately one month earlier than they would appear if printed. They are listed in the Table of Contents of the next open issue of JNeurosci. Cite this article as: JNeurosci, 1999, 0:RC13 (1-7). The publication date is the date of posting online at www.jneurosci.org.

http://www.jneurosci.org/cgi/content/full/3143 
$\mathrm{Ca}^{2+}$-insensitive and used to view basal fluorescence), $92 \mathrm{KMeSO}_{4}, 20$ $\mathrm{NaCl}, 5 \mathrm{Mg}$-ATP, $0.3 \mathrm{GTP}, 10 \mathrm{HEPES}$, and $0.3-0.8 \%$ biocytin $\mathrm{HCl}, \mathrm{pH}$ 7.3 (280 mOsM, adjusted with $\mathrm{KMeSO}_{4}$ ). After neurons were loaded, the electrode was removed, and the cells were allowed to recover in the presence of $0.3 \mu \mathrm{M}$ tetrodotoxin (TTX) containing saline for 1-2 hr. The concentration of fluo-3 loaded into the cell was estimated to be $300-500$ $\mu \mathrm{M}$. The following extracellular solution was used to measure the $\mathrm{Ca}^{2+}$ component of mEPSCs (in mM): $137 \mathrm{NaCl}, 5 \mathrm{KCl}, 5 \mathrm{CaCl}_{2}, 0 \mathrm{MgCl}_{2}$, $0.34 \mathrm{Na}_{2} \mathrm{HPO}_{4}\left(7 \mathrm{H}_{2} \mathrm{O}\right), 10$ Na-HEPES, 22 glucose, $1 \mathrm{NaHCO}_{3}, 0.02$ picrotoxin, and 0.0003 TTX, pH 7.4 (Murphy et al., 1994).

Imaging and analysis. Imaging was performed with a $100 \times 1.3 \mathrm{NA}$ Zeiss (Thornwood, NY) objective on a Zeiss Axiovert microscope with a stage that permitted movement during patch-clamp recording. A fiberoptically coupled intensified CCD camera with a Gen III intensifier tube was used for image acquisition (33 msec temporal resolution; Stanford Photonics, Palo Alto CA) with an Epix (Northbrook, IL) 4M12-64 MB frame grabber. For each experiment, 300 fluo-3 images were collected in $10 \mathrm{sec}$ sweeps (15-17 sweeps per experiment) and were analyzed off-line in Interactive Data Language (Research Systems Inc., Boulder, CO). To view processes under baseline conditions and to correct for process volume and cell loading (see below), the fluorescent $\mathrm{Ca}^{2+}$ indicator furaptra (excitation $380 \mathrm{~nm}$ ) was co-injected with fluo-3. We have used fluo- 3 in combination with furaptra because we find the affinity of the calcium indicator fura-2 is too high to accurately measure MSCTs. Our experiments indicate that fluo-3 provides better dynamic range and signal linearity than fura-2. These unpublished experiments were performed by examining the linearity of the dendritic $\mathrm{Ca}^{2+}$ signal in response to increasing numbers of action potentials. A single furaptra image (average of 30 images acquired over $1 \mathrm{sec}$ ) was taken for every trial after $10 \mathrm{sec}$ of fluo-3 data acquisition. We then placed measurement boxes, which were identical to those used for fluo-3 measurements, over a spine or dendrite of interest. The furaptra signal was corrected for autofluorescence by subtracting the background pixel value adjacent to a dendritic region. Background fluorescence was highly stable and did not exhibit changes that resemble those during MSCTs. By keeping the soma of the cell (a source of unbleached dye) out of the illumination path of the microscope we were able to minimize photobleaching. Photobleaching was minimal, because we observed very little change in furaptra fluorescence over consecutive trials, and this change was not progressive. Furaptra was chosen for its high fluorescence signal at basal $\mathrm{Ca}^{2+}$, its low affinity for $\mathrm{Ca}^{2+}$ with little attendant buffering, and its distinctive spectra that did not contaminate fluo-3 signals. For presentation purposes (and not quantification) the gray scale images in Figures 1 and 3 were deconvolved using an iterative routine based on a Gaussian approximation of the point spread function of the microscope used. $\mathrm{Ca}^{2+}$ signals were considered MSCTs if at least four consecutive fluorescence measurements were $1 \mathrm{SD}$ above baseline fluorescence; the initiation time was defined by the first point above baseline. The initiation site of the MSCT was identified by the earliest rise in fluorescence. In almost all cases, the site of MSCT initiation was associated with a clear morphological feature such as a varicosity or spine. $\mathrm{Ca}^{2+}$ responses were calculated for individual trials within $1.4-2.0 \mu \mathrm{m}^{2}$ regions surrounding sites of MSCT origin (spines). One complication with using $\mathrm{Ca}^{2+}$ imaging to measure synaptic responses is that because spines differ in size, the amount of $\mathrm{Ca}^{2+}$ flux and not necessarily the intraspine compartment concentration of $\mathrm{Ca}^{2+}$ reflects the level of NMDA receptor activity and mEPSC amplitude. We therefore quantified $\mathrm{Ca}^{2+}$ responses in two ways: first, raw change in fluo-3 fluorescence $\left(\Delta F_{480}\right)$; and second, change in fluo-3 fluorescence scaled to spine volume $\left(\Delta F_{480} / F_{380}\right.$; basal furaptra fluorescence). The first measure, $\Delta F_{480}$, was assumed to be directly proportional to $\mathrm{Ca}^{2+}$ flux $\left(I_{\mathrm{NMDA}}\right)$, because our experiments use a high concentration of fluo- $3 \mathrm{Ca}^{2+}$ indicator, which is likely to be the dominant cellular $\mathrm{Ca}^{2+}$ buffer and will therefore efficiently capture incoming $\mathrm{Ca}^{2+}$ and indicate flux (Neher, 1995). However, dendrites of cultured neurons exhibit an irregular profile along the $z$-axis (of focus). If absolute measures of fluorescence intensity are used, suboptimal focus will lead to errors in quantification. To provide a correction for suboptimal focus, we have included a second low-affinity calcium probe in the pipette solutionfuraptra $F_{380}$ (insensitive to MSCT $\mathrm{Ca}^{2+}$ fluxes). In this case observed fluorescence values of both $\Delta F_{480} \alpha I_{\mathrm{NMDA}}$ and $\mathrm{F}_{380} \alpha$ volume (vol; see below) were reduced by a factor proportional to $F_{380} / F_{380 \max }$, the ratio of the observed $F_{380}$ to a theoretical maximal in focus $F_{380 \max }$ (see Eqs. 1, 2). Therefore, for the data shown we used the second method of quantification, $\Delta F_{480} / F_{380}$, which substantially reduced errors attributable to focal differences (our unpublished results) by dividing a measure of raw
$\mathrm{Ca}^{2+}$ influx $\left(\Delta F_{480}\right)$ by a measure of spine size $\left(F_{380}\right)$. By using the fluorescence ratio (a measure of $\mathrm{Ca}^{2+}$ concentration), we did not need to determine the actual in focus values of $\Delta F_{480}$ and $F_{380}$ (see Eq. 3). Because larger spines have a greater volume and hence larger $F_{380}$, the use of $\Delta F_{480} / F_{380}$ ratios would cause us to underestimate, rather than account for, observed differences in $\mathrm{Ca}^{2+}$ response $\left(I_{\mathrm{NMDA}}\right)$ between spines of different volume (see Eq. 4, Fig. 2a, dashed line). For example, if all spines had identical $\Delta F_{480} / F_{380}$ ratios (and thus $\mathrm{Ca}^{2+}$ concentration) during MSCTs, then the larger spines must have had an NMDA current that was of relatively higher amplitude (Eqs. 3, 4). Similar correlations between synapse size and response amplitude were observed with both methods of measurement (data not shown). Relative $\mathrm{Ca}^{2+}$ transient amplitude (scaled to mean) was used to calculate correlations between $\mathrm{Ca}^{2+}$ response amplitude and synapse size, because differences in the absolute calibration of the $\Delta F_{480} / F_{380}$ ratios between the specimens would artifactually increase the variability of correlations. Using other methods of normalizing the MSCT amplitude (scaling to median or maximum response) did not appreciably alter the correlations that were observed. To examine the correlation between measures of synapse size and responsiveness, we pooled data from multiple neurons to obtain higher statistical power. Although lacking statistical power, analysis of single experiments indicated that all four EM samples showed a positive correlation between the $\Delta F_{480} / F_{380}$ ratios and spine volume (indicating a trend toward significance). None of the samples showed a negative correlation.

$$
\begin{gathered}
\Delta F_{480} \alpha I_{\mathrm{NMDA}} *\left(F_{380} / F_{380 \max }\right) \\
F_{380} \alpha \operatorname{vol} *\left(F_{380} / F_{380 \max }\right) ; \\
\Delta F_{480} / F_{380} \alpha I_{\mathrm{NMDA}} / \mathrm{vol} ; \\
I_{\mathrm{NMDA}} \alpha\left(\Delta F_{480} / F_{380}\right) * \mathrm{vol} .
\end{gathered}
$$

Spine size was scaled to the average spine size observed in a particular specimen to control for potential differences in spine size attributable to fixation conditions, development, or phenotype of neuron (Papa et al., 1995; Boyer et al., 1998). We have also used other methods of calculating relative spine size (scaled to median or maximum spine volume) and have found that the correlations do not vary significantly with the method used.

Electron microscopy. After MSCT imaging, preparations were fixed with $4 \%$ paraformaldehyde and $0.2-0.5 \%$ glutaraldehyde in 0.1 м Sörensen's $\mathrm{Na}^{+}$phosphate buffer, $\mathrm{pH} 7.2-7.4(1.5 \mathrm{hr})$ at room temperature (RT), rinsed briefly in Dulbecco's PBS (DPBS), permeabilized in 0.1$0.2 \%$ Triton X-100 in DPBS (3-4 min, RT), washed with DPBS (3-5 vol over 5 min, RT) and blocked in $2.5 \%$ normal goat serum in DPBS (4-12 $\left.\mathrm{hr}, 4^{\circ} \mathrm{C}\right)$. Specimens were then washed with DPBS (3-5 vol over $30 \mathrm{~min}$, RT), incubated with Vector Laboratories (Burlingame, CA) A/B reagent (avidin/biotinylated peroxidase complex; $1 \mathrm{hr}, \mathrm{RT}$ ), washed with DPBS (3-5 vol over $30 \mathrm{~min}, \mathrm{RT}$ ), and incubated in $0.5 \mathrm{mg} / \mathrm{ml}$ diaminobenzidine (DAB) and $0.015 \% \mathrm{H}_{2} \mathrm{O}_{2}$ in DPBS for $2-5 \mathrm{~min}$ (RT, intensity monitored to prevent overstaining). DPBS washing (5 vol over at least $30 \mathrm{~min}, \mathrm{RT}$ ) was followed by further fixation in $2.5 \%$ glutaraldehyde in 0.1 M Sörensen's buffer, $\mathrm{pH}$ 7.2-7.4 (1 hr, on ice) and washing in the same buffer (3 vol over 30 min, on ice). Preparations were then post-fixed in $1 \% \mathrm{OsO}_{4}$ in the same buffer (1 hr, on ice). After a final Sörensen's buffer wash (3 vol over $30 \mathrm{~min}$, on ice), cultures were dehydrated in a graded ethanol series $(50,70,85,95$, and 100\%) and flat-embedded in Spurr resin on Aclar plastic (Proplastics, Linden, NJ). After polymerization, areas containing single stained neurons were excised, separated from the Aclar, and mounted on blank blocks. Serial sections of $\sim 70 \mathrm{~nm}$ thickness were collected on pioloform or Formvar-coated single slot grids, stained with $3 \%$ aqueous uranyl acetate (UA) or $5 \%$ UA in $20 \% \mathrm{MeOH}$, followed by lead citrate, and then examined at $80 \mathrm{keV}$ in a Zeiss EM 10C serial transmission electron microscope. A montage of low-power electron micrographs $(8000 \times)$ was aligned with fluorescence and bright-field images. From this overlay, it was possible to identify DAB-stained dendritic spines where MSCT events occurred. Staining selectivity arises because both the $\mathrm{Ca}^{2+}$ indicators and biocytin are injected into a single neuron, allowing the $\mathrm{Ca}^{2+}$ imaging and subsequent staining of the neuron of interest with an immunoperoxidase reaction (Gulyás et al., 1993). EM images were obtained at higher magnification $(31,500 \times)$ to perform serial reconstruction at spines where MSCT events were initiated. Spine volume and synaptic contact area were measured at sites with more than one MSCT by tracing the outline of DAB-stained spines through serial sections and digitizing the traces. NIH Image and Adobe 
(Mountain View, CA) Photoshop were used for three-dimensional reconstructions. Profiles were occasionally unobtainable because of section folding. In such cases, spine sizes were estimated from the digitized serial sections by linear interpolation between adjacent sections; data were used only if the amount estimated accounted for $<10 \%$ of spine volume. The addition of estimated data did not appreciably change the correlations that were obtained. In some specimens detergents required in the staining process degraded the ultrastructure.

Synapses were identified by the presence of presynaptic and postsynaptic membrane apposition, synaptic cleft thickening, a presynaptic paramembranous density, and clustering of at least three vesicles near the presynaptic membrane. The intensity of the DAB staining prevented accurate measurement of the postsynaptic density size in many cases. The area of synaptic contact was defined as the region of increased (and relatively constant) thickness between the presynaptic and postsynaptic membranes, as illustrated in Figure 1d, arrows. Measurements of synaptic contact area in perforated synapses (synapses with two separate clusters of vesicles from the same presynaptic bouton) included the area of the perforation. Because relative spine size (scaled to the mean spine size for each specimen) was correlated with MSCT amplitude (also scaled to mean MSCT amplitude for each specimen), no correction was applied for differential shrinkage in the $z$-dimension during ethanol dehydration (Trommald and Hullenberg, 1997). Twenty synapses were reconstructed from four specimens (neurons). Of these, 2 were shaft synapses; spine volume was therefore measured at the remaining 18 spines (12 single macular synapses, 4 perforated synapses with the same presynaptic bouton, and 2 spines each contacting 2 presynaptic boutons). At 7 of the 20 synapses, the synaptic contact area was not measurable throughout its full extent because of a tangential plane of section. Synaptic contact area was therefore measured at 13 of the 20 synapses; this included two of the perforated synapses but none of the synapses with more than one presynaptic bouton.

Confocal microscopy. After fluorescence imaging of MSCTs using wide-field microscopy, cells were fixed with $4 \%$ paraformaldehyde in 0.1 M Sörensen's buffer, $\mathrm{pH}$ 7.2-7.4 (1.5 hr, RT), rinsed briefly in DPBS, permeabilized in $0.2-0.5 \%$ Triton $\mathrm{X}-100$ in DPBS (4-5 min, RT), washed with DPBS (3-5 vol over 5 min, RT), and blocked in 1.5-2.5\% normal goat serum in DPBS $\left(4-12 \mathrm{hr}, 4^{\circ} \mathrm{C}\right)$. Cultures were washed with DPBS (3-5 vol over $30 \mathrm{~min}, \mathrm{RT}$ ), incubated with $20 \mu \mathrm{g} / \mathrm{ml}$ avidinfluorescein in DPBS (Vector; 1-2 hr, RT), washed with DPBS (3-5 vol over $30 \mathrm{~min}, \mathrm{RT}$ ), and mounted on a coverslip with Antifade in glycerol and DPBS (Molecular Probes, Eugene, OR). Confocal imaging was performed with a Bio-Rad (Hercules, CA) MRC 600 system attached to a Zeiss Axioskop microscope and a $100 \times 1.3$ NA Zeiss objective (laser intensity $=1 \%$; confocal pinhole $=3$ Bio-Rad units). Serial images along the vertical axis ( $z$-series) were obtained through the entire dendritic region of interest (step size, $0.54 \mu \mathrm{m}$ ) and a maximal-intensity projection was used to generate a two-dimensional representation of spine size. A maximal-intensity projection flattens 3-D images by creating an image of the maximal pixel value across the sections for each pixel. For a spine to be selected for measurement, it was necessary to clearly resolve at least one MSCT event initiated at the spine. MSCT imaging was performed with wide-field microscopy and a CCD camera that has lower resolution than confocal microscopy. This criterion was the limiting factor in spine determination; therefore, other morphological criteria were not necessary (Trommald et al., 1995). Spines that were selected could be clearly resolved from other structures in the maximal-intensity confocal projection image; at least two rows of pixels of lower intensity were between two adjacent spines or between spine head and dendrite. Cross-sectional spine area was quantified using NIH Image and Adobe Photoshop. Although confocal measurements of absolute spine size are problematic for small spines (Harris, 1994; Trommald and Hullenberg, 1997), a correction was not applied, because relative measurements of spine size versus MSCT response were used. Because of the limiting resolution of confocal microscopy, confocal measurements of spine size are likely to overestimate the size of small spines; this effect would underestimate rather than account for a correlation between spine size and MSCT amplitude.

\section{RESULTS}

\section{Calcium imaging and parallel ultrastructural analysis of single synapses}

We have conducted experiments designed to assess both structure and function at the same CNS synapses. Cultured cortical neu- rons were injected with fluo-3, and MSCT imaging was used to map the NMDA receptor-mediated component of quantal synaptic responses to identified dendritic regions. Co-injection of biocytin allowed the selective staining of the neuron of interest after MSCT imaging. Serial EM reconstruction was performed on 20 synapses (four neurons) from which we had measured the postsynaptic effect of putative single transmitter quanta. Figure $1 a$ shows a basal fluorescence image of a region of dendrite from a cortical neuron. Figure $1 b$ illustrates traces of $\mathrm{Ca}^{2+}$ dynamics at four dendritic sites. A perforated synapse was identified at site 3 , where seven synaptic events were initiated. In contrast, most neighboring dendritic synapses were either inactive (for example, site 4) or were the initiation site of only one MSCT event. Sites 1 and 2 represent other sites, subsequently confirmed by serial reconstruction to be single macular synapses, with smalleramplitude responses. MSCTs were usually localized to dendritic spines, as illustrated by a sequence of images during a single trial of a $\mathrm{Ca}^{2+}$ transient initiated at site 3 (Fig. 1c). After $\mathrm{Ca}^{2+}$ imaging, specimens containing the dendritic region of interest were identified at both the light and EM levels via immunoperoxidase staining (Fig. 1d). EM revealed a single large perforated spine synapse centered at site 3 (Fig. 1d,e). Analysis of serial sections indicated that adjacent synapses were more than $2 \mu \mathrm{m}$ away. Given the point spread function of the microscope (which describes the attenuation of signal with distance), it is unlikely that fluorescence changes at other synapses contributed significantly to the events measured at this site (our unpublished observations). Figure $1 e$ shows a view of the 3-D reconstruction of site 3 made from serial EM sections, confirming the existence of a large dendritic spine with a single perforated synapse. The combined use of MSCT imaging and serial EM reconstruction thus enables the comparison of NMDA-mediated quantal responses at morphologically identified CNS synapses.

\section{The amplitude of the miniature synaptic calcium transient is correlated with synapse size}

Spontaneous quantal synaptic currents exhibit a heterogeneous amplitude distribution that cannot be fully attributed to spatial distribution and filtering (Manabe et al., 1992; Lisman and Harris, 1993) but may be attributed to difference in response amplitude between synapses. The amplitude distribution may reflect differences in the size of the postsynaptic densities (PSDs), a hypothesis that we sought to test via combined $\mathrm{Ca}^{2+}$ imaging and serial reconstruction EM. Because we were often unable to measure the size of the postsynaptic density because of the intensity of the DAB staining, we measured two other variables that are correlated with PSD size: spine volume and the area of synaptic contact (Harris and Stevens, 1989; Harris and Sultan, 1995; Trommald and Hullenberg, 1997). Both measures of synapse size were significantly positively correlated $(p<0.05)$ with the NMDA receptor-mediated component of the quantal response amplitude (Fig. 2). Figure $2 a$ indicates a significant positive correlation between relative spine volume and relative MSCT amplitude $(r=0.51 ; p<0.05)$. The gray dashed line indicates the predicted relationship between spine volume and MSCT amplitude if no relationship existed between $I_{\mathrm{NMDA}}$ and spine volume, that is, for a constant $I_{\mathrm{NMDA}}$ (see Eq. 3, Materials and Methods). A significant positive correlation was also observed between relative synaptic contact area and relative MSCT amplitude $(r=$ 0.75 ; Fig. $2 b$ ). The difference between the correlation coefficents in Figure 2, $a$ and $b$, may be attributable to chance (given the scatter in the correlations) or may be because synaptic contact 

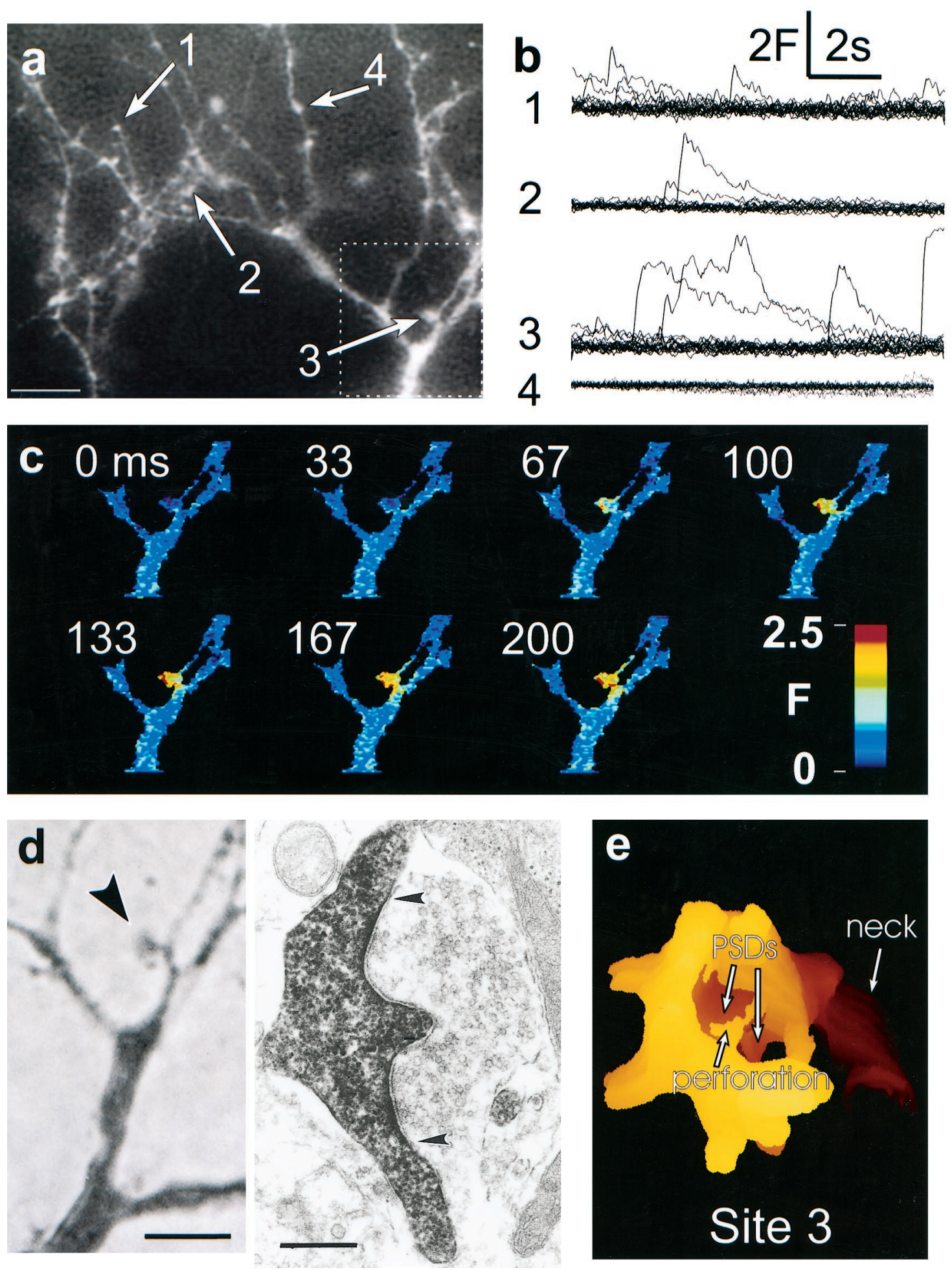

Figure 1. Quantal synaptic activity and serial reconstruction of identified cortical synapses. $a$, Basal furaptra fluorescence image $\left(F_{380}\right.$; Ca ${ }^{2+}$ independent excitation wavelength) of a region of dendrite where $\mathrm{Ca}^{2+}$ dynamics were measured. Scale bar, $10 \mu \mathrm{m} . b$, Plots of $\mathrm{Ca}^{2+}$ response versus time at four dendritic sites (17 trials $10 \mathrm{sec}$ in length are overplotted). MSCTs were initiated and measured at $2 \mu \mathrm{m}^{2}$ regions centered over the indicated sites. Units of fluorescence: $F=$ change in fluo-3 fluorescence/basal furaptra fluorescence, $\Delta F_{480} / F_{380}$. Site 3 exhibited a higher MSCT frequency and larger average MSCT amplitude than other sites, whereas site 4 showed no activity. $c$, Images of the initiation of an MSCT at 33 msec intervals in the dendritic region encompassing site 3 (in $a, b$ ). In this trial, an MSCT is first visible at $67 \mathrm{msec} . d$, Left panel, Bright-field image of the same region of dendrite after fixation and immunoperoxidase staining. The arrow points to site 3 shown above. Scale bar, $5 \mu \mathrm{m}$. Right panel, Electron micrograph illustrating a single cross-section through the spine at site 3 . The arrowheads demarcate the region of cleft thickening used to measure synaptic contact area. On the left of the image, the DAB-stained spine is visible. A single perforated synapse is visible. Scale bar, $0.4 \mu \mathrm{m}$. $e$, Three-dimensional view of reconstruction of the spine at site 3 confirms the presence of a single perforated synapse onto a large spine head (postsynaptic region only is shown). Examination of $>40$ serial sections through site 3 indicated only one synapse. The postsynaptic densities, the perforation, and the spine neck are identified by arrows. PSDs were identified from lighter prints of EM images. 


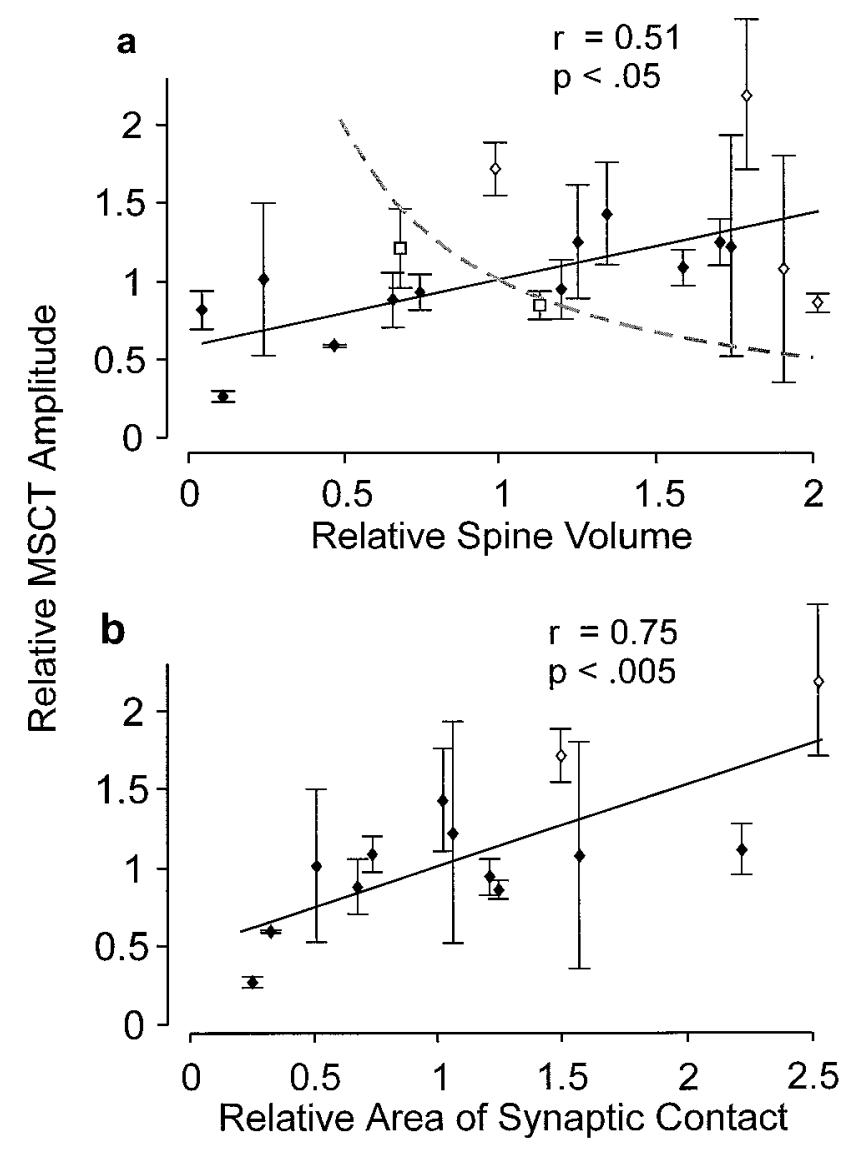

Figure 2. Significant positive correlation between quantal amplitude and two measures of synapse size. Quantal amplitude was measured as the average MSCT amplitude at sites with repeated MSCT events. MSCT amplitudes \pm SEM scaled to the mean of each experiment were plotted versus two measures of synapse size (see below). $a$, Spine volume (vs MSCT amplitude) determined from serial reconstruction of 18 spines that were repeated MSCT initiation sites. The gray dashed line indicates the expected relationship between MSCT amplitude and spine volume for a constant $I_{\mathrm{NMDA}}$. $\diamond$, Spines with one macular synapse; $\diamond$, spines with one perforated synapse; $\square$, spines with two presynaptic inputs. $b$, Area of synaptic contact (vs MSCT amplitude) was measured from serial EM reconstructions of 13 synapses (see Materials and Methods). $\downarrow$, Macular synapse; $\diamond$, perforated synapse.

area is likely better correlated with postsynaptic density size. To test whether three synapses with high SEM (because of few events) could spuriously account for the significant correlations observed, these data points were removed from the analysis, resulting in a slightly higher correlations. Thus, the behavior of the highly variable synapses did not account for the significant correlations between measures of synaptic size and MSCT amplitude.

\section{Confocal measurement of spine size correlates with miniature $\mathrm{Ca}^{2+}$ transient amplitude}

To confirm with a larger data set the observed relationship between synapse size as measured by serial EM reconstruction and MSCT amplitude, we performed similar experiments using confocal microscopy to measure spine size. In these experiments neurons were also injected with a combination of biocytin and $\mathrm{Ca}^{2+}$ indicators, and MSCT imaging was performed under the same conditions as described above. Figure $3 a$ shows a basal fluorescence image of a region of dendrite captured using wide-


Figure 3. Significant correlation between quantal amplitude and spine size measured with confocal microscopy. $a$, Wide-field basal fluorescence $\left(F_{380}\right)$ image of a region of cortical neuron dendrite. Arrows indicate three sites where MSCTs were initiated. After wide-field MSCT imaging, specimens were fixed and stained with avidin-fluorescein. $b$, Maximalintensity projection (see Materials and Methods) of the same region of dendrite stained with avidin-fluorescein obtained from a stack of confocal optical sections. The three spines are labeled with arrows and are clearly resolved from the background. Scale bar, $10 \mu \mathrm{m}$. $c$, Traces of $\mathrm{Ca}^{2+}$ response versus time at the three dendritic spines indicated in $a$ and $b$. Traces were averages of two to six MSCTs and were aligned to the time of first rise in $\mathrm{Ca}^{2+} . d$, MSCT amplitude plotted versus spine size (scaled to the mean of each experiment) for 74 spines from five cells; $p<10^{-5}$.

field microscopy and a CCD camera. After imaging, specimens were fixed and stained with avidin-fluorescein, and confocal measurements of spine size were performed. Figure $3 b$ shows a confocal image of the region of dendrite. Figure $3 c$ illustrates the average MSCT $\mathrm{Ca}^{2+}$ response at three spines, each of which was a site of repeated MSCT initiation. As with the EM data, a significant positive correlation was observed between spine size and $\mathrm{Ca}^{2+}$ transient amplitude in 74 spines from five neurons (Fig. $\left.3 d ; r=0.52 ; p<10^{-5}\right)$.

\section{DISCUSSION}

It has been widely hypothesized that alterations in synapse structure underlie changes in synapse efficacy (Lisman and Harris, 1993; Edwards, 1995). Many experiments have reported structural changes in synaptic populations after manipulations of synaptic strength or after learning (Greenough et al., 1978; Fifkova et al., 1982; Desmond and Levy, 1988; Hosokawa et al., 1995; Papa and Segal, 1996; Moser et al., 1997; Rusakov et al., 1997; but see Sorra and Harris, 1998). Unlike population studies, we have directly compared structure and function at the same synapses. This is the first report comparing the functional and structural properties of multiple synapses within the same CNS neuron, although previous investigations have obtained functional data from single 
synapses that were later investigated at the ultrastructural level (Gulyás et al., 1993; Buhl et al., 1994, 1997). We conclude that the NMDA receptor-mediated component of quantal size (as measured by $\mathrm{Ca}^{2+}$ influx) is correlated with the size-related parameters of spine volume and synaptic contact area.

We have used $\mathrm{Ca}^{2+}$ imaging in cultured cortical neurons to measure the localized $\mathrm{Ca}^{2+}$ component of the miniature synaptic response (the MSCT). Under these conditions, the average MSCT amplitude provides a measure of the average mEPSC amplitude (attributed to NMDA receptors) of an identified synapse for the following reasons. First, synapses are confirmed to be present at each site of MSCT initiation, strongly suggesting that the $\mathrm{Ca}^{2+}$ influx is of synaptic origin. Second, the amplitude of the MSCT is correlated with the amplitude of the underlying mEPSC (Murphy et al., 1995). Third, both MSCTs and the slow component of the mEPSC are blocked in the presence of the NMDA receptor antagonist APV (Murphy et al., 1994, 1995).

We observed that spine size and the area of synaptic contact are significantly correlated with MSCT amplitude. Additional experiments using confocal rather than EM spine measurement confirmed these findings. These results support the hypothesis that larger synapses show larger quantal responses (Harris and Stevens, 1989; Lisman and Harris, 1993), although it is important to stress that the current MSCT imaging method measures the NMDA receptor-mediated component of the quantal response, and thus no conclusions can be made about the AMPA receptor mediated component. Although we observed significant correlations between size and MSCT amplitude, the considerable scatter in the correlations suggests that other factors may also be contributing to MSCT size, including differences in vesicular transmitter content, and in the stochastic properties of postsynaptic receptors (Frerking et al., 1995; Murphy et al., 1995; Auger and Marty, 1997; Nusser et al., 1997). Additionally, multivesicular release may have contributed to the variability (Auger et al., 1998; Prange and Murphy, 1999). Furthermore, it is conceivable that fixation conditions may have slightly altered the synapse contact area or morphology. A further possibility is that $\mathrm{Ca}^{2+}$ release from intracellular stores may have amplified MSCT amplitudes, as recently reported by Emptage et al. (1999). However, our previous results (Murphy et al., 1995) indicated a strong correlation between $\mathrm{Ca}^{2+}$ transient amplitude and the amplitude of miniature excitatory synaptic current at a particular synapse. Therefore, if $\mathrm{Ca}^{2+}$ release from stores does amplify our optical signals, they are nonetheless proportional to synaptic current amplitude.

Although we were unable to measure directly the PSD area in our study, other measures of synapse size were significantly correlated with response amplitude, suggesting that PSD size is also correlated with NMDA receptor-dependent quantal amplitude. The strongest correlation with MSCT amplitude was observed for the measurement of synaptic contact area (Fig. $2 b$ ). Given the scatter in the correlations, this difference may have been attributable to chance; alternatively, this difference may reflect a stronger relationship between synaptic contact area and postsynaptic density size. Larger synaptic contacts and thus PSDs may contain a greater number of functional receptors (Harris and Landis, 1986), suggesting a functional consequence of activity-dependent regulation of PSD composition (Rao and Craig, 1997). In cerebellar stellate cells, postsynaptic $\mathrm{GABA}_{\mathrm{A}}$ receptor density is uniform (Nusser et al., 1997), suggesting that synapse size may be a reliable measure of receptor number. In CA1 of hippocampus, although the density of AMPA receptors may not be constant,
AMPA receptor immunoreactivity is greater at larger spines than at smaller spines (Nusser et al., 1998). We are not aware of any reports correlating NMDA receptor density and synapse size. Given the recent identification of relatively complex protein arrays involved in clustering postsynaptic receptors (Sheng, 1997), PSD area may be a limiting factor in controlling receptor expression (Kennedy, 1997). We conclude that mechanisms that control the growth and elaboration of synapses are thus likely to regulate NMDA receptor-mediated quantal amplitude.

\section{REFERENCES}

Auger C, Marty A (1997) Heterogeneity of functional synaptic parameters among single release sites. Neuron 19:139-150.

Auger C, Kondo S, Marty A (1998) Multivesicular release at single functional synaptic sites in cerebellar stellate and basket cells. J Neurosci 18:4532-4547.

Boyer C, Schikorski T, Stevens CF (1998) Comparison of hippocampal dendritic spines in culture and in brain. J Neurosci 18:5294-5300.

Buhl EH, Halasy K, Somogyi P (1994) Diverse sources of hippocampal unitary inhibitory potentials and the number of synaptic release sites. Nature 368:823-828.

Buhl EH, Tamás G, Szilágyi T, Stricker C, Paulsen O, Somogyi P (1997) Effect, number and location of synapses made by single pyramidal cells onto aspiny interneurones of cat visual cortex. J Physiol (Lond) 500:689-713.

Calverley RKS, Jones DG (1990) Contributions of dendritic spines and perforated synapses to synaptic plasticity. Brain Res Rev 15:215-249.

Desmond NL, Levy WB (1988) Synaptic interface surface area increases with long-term potentiation in the hippocampal dentate gyrus. Brain Res 453:308-314.

Edwards FA (1995) Anatomy and electrophysiology of fast central synapses lead to a structural model for long-term potentiation. Physiol Rev 75:759-787.

Emptage N, Bliss TV, Fine A (1999) Single synaptic events evoke NMDA receptor-mediated release of calcium from internal stores in hippocampal dendritic spines. Neuron 22:115-124.

Fifkova E, Anderson CL, Young SJ, van Harrevald A (1982) Effect of anisomycin on stimulation-induced changes in dendritic spines of the dentate granule cells. J Neurocytol 11:183-210.

Frerking M, Borges S, Wilson M (1995) Variation in GABA mini amplitude is the consequence of variation in transmitter concentration. Neuron 15:885-895.

Greenough WT, West RW, DeVoogd TJ (1978) Subsynaptic plate perforations: changes with age and experience in the rat. Science 202:1096-1098.

Gulyás AI, Miles R, Sik A, Tóth K, Tamamaki N, Freund TF (1993) Hippocampal pyramidal cells excite inhibitory neurons through a single release site. Nature 366:683-686.

Harris KM (1994) Serial electron microscopy as an alternative or complement to confocal microscopy for the study of synapses and dendritic spines in the central nervous system. In: Three-dimensional confocal microscopy: volume investigation of biological specimens (Stevens JK, Mills LR, Trogladis JE, eds), pp 421-445. New York: Academic.

Harris KM, Landis DMD (1986) Synaptic membrane structure in area CA1 of the rat hippocampus. Neuroscience 19:857-872.

Harris KH, Stevens JK (1989) Dendritic spines of CA1 pyramidal cells in the rat hippocampus: serial electron microscopy with reference to their biophysical characteristics. J Neurosci 9:2982-2997.

Harris KM, Sultan P (1995) Variation in the number, location and size of synaptic vesicles provides an anatomical basis for the nonuniform probability of release at hippocampal CA1 synapses. Neuropharmacology 34:1387-1395.

Harris KM, Jensen FE, Tsao B (1992) Three-dimensional structure of dendritic spines and synapses in rat hippocampus (CA1) at postnatal day 15 and adult ages: implications for the maturation of synaptic physiology and long-term potentiation. J Neurosci 12:2685-2705.

Hosokawa T, Rusakov DA, Bliss TVP, Fine A (1995) Repeated confocal imaging of individual dendritic spines in the living hippocampal slice: evidence for changes in length and orientation associated with chemically induced LTP. J Neurosci 15:5560-5573.

Kennedy MB (1997) The postsynaptic density at glutamatergic synapses. Trends Neurosci 20:264-268.

Lisman JE, Harris KM (1993) Quantal analysis and synaptic anatomy- 
integrating two views of hippocampal plasticity. Trends Neurosci 16:141-147.

Mackenzie PJ, Umemiya M, Murphy TH (1996) $\mathrm{Ca}^{2+}$ imaging of CNS axons in culture indicates reliable coupling between single action potentials and distal functional release sites. Neuron 16:783-795.

Manabe T, Renner P, Nicoll RA (1992) Postsynaptic contribution to long-term potentiation revealed by the analysis of miniature synaptic currents. Nature 355:50-55.

Moser M-B, Trommald M, Egeland T, Andersen P (1997) Spatial training in a complex environment and isolation alter the spine distribution differently in rat CA1 pyramidal cells. J Comp Neurol 380:373-381.

Murphy TH, Baraban JM, Wier WG, Blatter LA (1994) Visualization of quantal synaptic transmission by dendritic calcium imaging. Science 263:529-532.

Murphy TH, Baraban JM, Wier WG (1995) Mapping miniature synaptic currents to single synapses using calcium imaging reveals heterogeneity in postsynaptic output. Neuron 15:159-168.

Neher E (1995) The use of fura-2 for estimating $\mathrm{Ca}^{2+}$ buffers and $\mathrm{Ca}^{2+}$ fluxes. Neuropharmacology 34:1423-1442.

Nusser Z, Cull-Candy S, Farrant M (1997) Differences in synaptic GABA(A) receptor number underlie variation in GABA mini amplitude. Neuron 19:697-709.

Nusser Z, Lujan R, Laube G, Roberts JDB, Molnar E, Somogyi P (1998) Cell type and pathway dependence of synaptic AMPA receptor number and variability in the hippocampus. Neuron 21:545-559.

Papa M, Segal M (1996) Morphological plasticity in dendritic spines of cultured hippocampal neurons. Neuroscience 71:1005-1011.

Papa M, Bundman MC, Greenberger V, Segal M (1995) Morphological analysis of dendritic spine development in primary cultures of hippocampal neurons. J Neurosci 15:1-11.

Petrozzino JJ, Pozzo Miller LD, Connor JA (1995) Micromolar Ca2+ transients in dendritic spines of hippocampal pyramidal neurons in brain slice. Neuron 14:1223-1231.
Pierce JP, Lewin GR (1994) An ultrastructural size principle. Neuroscience 58:441-446.

Prange O, Murphy TH (1999) An analysis of multiquantal transmitter release from single cultured cortical neuron terminals. J Neurophysiol 81:1810-1817.

Rao A, Craig AM (1997) Activity regulates the synaptic localization of the NMDA receptor in hippocampal neurons. Neuron 19:801-812.

Rusakov DA, Davies HA, Harrison E, Diana G, Richter-Levin G, Bliss TV, Stewart MG (1997) Ultrastructural synaptic correlates of spatial learning in rat hippocampus. Neuroscience 80:69-77.

Schikorski T, Stevens CF (1997) Quantitative ultrastructural analysis of hippocampal excitatory synapses. J Neurosci 17:5858-5867.

Schiller J, Schiller Y, Clapham DE (1998) NMDA receptors amplify calcium influx into dendritic spines during associative pre- and postsynaptic activation. Nat Neurosci 1:114-118.

Sheng M (1997) Excitatory synapses. Glutamate receptors put in their place. Nature 386:221-223.

Sorra KE, Harris KM (1998) Stability in synapse number and size at 2 $\mathrm{hr}$ after long-term potentiation in hippocampal area CA1. J Neurosci 18:658-671.

Trommald M, Hullenberg G (1997) Dimensions and density of dendritic spines from rat dentate granule cells based on reconstructions from serial electron micrographs. J Comp Neurol 377:15-28.

Trommald M, Jensen V, Andersen P (1995) Analysis of dendritic spines in rat CA1 pyramidal cells intracellularly filled with a fluorescent dye. J Comp Neurol 353:260-274.

Yuste R, Denk W (1995) Dendritic spines as basic functional units of neuronal integration. Nature 375:682-684.

Yuste R, Majewska A, Cash SS, Denk W (1999) Mechanisms of calcium influx into hippocampal spines: heterogeneity among spines, coincidence detection by NMDA receptors, and optical quantal analysis. J Neurosci 19:1976-1987. 